\title{
Overexpression of Stromal Interaction Molecule 1/Store-operated Calcium Entry-associated Regulatory Factor in Interstitial Cells of Cajal in Mouse Jejunum Impairs Pacemaker Activity
}

TO THE EDITOR: In a recent issue of Journal of Neurogastroenterology and Motility, Park et al ${ }^{1}$ examined the roles of stromal interaction molecule 1 (STIM1) and store-operated calcium entryassociated regulatory factor (SARAF) in pacemaker activity of interstitial cells of Cajal (ICCs) from mouse jejunum. Authors employed lipofectamine transfection to deliver STIM1, SARAF, and pcDNA vectors into the cultured ICC and evaluated the changes of pacemaker activities. The results show that the cultured ICCs are capable to produce spontaneously rhythmical pacemaker potentials or slow waves, which traces are very regular and rhythmical, robust and stabilized. For example about a 2-minute control trace the event frequency and amplitude are $14.7 \pm 0.8$ cycles/minute and -59.6 $\pm 2.6 \mathrm{mV}$. Compared with other renowned research groups, the amplitude of slow wave is about $23.0 \pm 1.6 \mathrm{mV}$ in whole cell configuration ${ }^{2}$ and $20.0 \mathrm{mV}$ using single channel driving force calculation. ${ }^{3}$ Based on such much better quality trace, Park et al ${ }^{1}$ applied modifications on it. Firstly, carbachol $(\mathrm{CCh})$ leads to trace baseline up and the slow waves diminished. It is meant that the activation of muscarinic (M2/M3) results in intracellular calcium ions $\left(\left[\mathrm{Ca}^{2+}\right] \mathrm{i}\right)$ increase through intracellular calcium stores, such as the endoplasmic reticulum (ER) and mitochondria. The increased intracellular calcium then consequently activates the calcium channel at the cellular membrane, therefore, $\mathrm{Ca}^{2+}$ influxes drastically into ICC causing ICC membrane potential depolarized with no repolarization, due to no enough efflux of $\mathrm{Ca}^{2+}$ to coordinate the slow waves. On the other hand, authors observed that there is no membrane potential depolarization accompanied pacemaker activity abolished under blockage of gadolinium $\left(\mathrm{Gd}^{+}\right)$or $\mathrm{Ca}^{2+}$-free condition. It implicates that without activation of M2/M3 and intracellular $\mathrm{Ca}^{2+}$ stores in ICC, the direct channel modification can stop calcium conductance but not remarkably alter the membrane potential. These results enhance that the slow wave or pacemaker activity is regulated through a mechanism called store-operated $\mathrm{Ca}^{2+}$ entry (SOCE), consistent with other reports. ${ }^{4,5}$

Since Torihashi et $\mathrm{al}^{6}$ first demonstrated the calcium oscillation linked to pacemaking of ICC in 2002, there have been many studies proceeded to understand how $\left[\mathrm{Ca}^{2+}\right] \mathrm{i}$ concentration alteration plays a clock like pacemaking function in ICC. ${ }^{7,8}$ However, to date, it is still unknown about how $\left[\mathrm{Ca}^{2+}\right] \mathrm{i}$ is regulated in ICCs. Park et al is the first to introduce $2 \mathrm{ER}$ proteins (STIM1/SARAF) in Journal of Neurogastroenterol Motil that is indeed intriguing us to have new insight into pacemaking mechanism of ICC.

Upon the observation of pacemaker activity expressed in ICC with overexpression of STIM1/SARAF, ${ }^{1}$ authors claim that STIM1 "enhances" the pacemaking activity by increasing the frequency of slow waves (ie, $33.6 \pm 6.2$ cycles $/ 2$ minutes in control versus to $48.6 \pm 5.2$ cycles $/ 2$ minutes in STIM1); whereas SARAF "inhibits" the pacemaking by decreasing the frequency (ie, $31.8 \pm 3.4$ cycles $/ 2$ minutes in control versus to $7.6 \pm 2.0$ cycles $/ 2$ minutes in SARAF). It seems that STIM1 facilitates the $\mathrm{Ca}^{2+}$ influx and SARAF facilitates the $\mathrm{Ca}^{2+}$ efflux, hence, both effects together are required to compromise to form up the rhythmically electrical pacemaker activity.

There are several arguments as follows: first, slow wave is named by its characterization of "slow" in which a relative wider duration or slow upstroke is the must for the intrinsic feature of ICC. Although there is no word of "slow wave" in article, the pacemaker potential should be no difference to "slow wave" here. Looking into the electrical waveforms of STIM1/SARAF, what mostly seen are spike-like events, in which some are mature and mostly are immature. It is seldom to see a regular waveform in traces except for both membrane potentials stay at around $-61 \mathrm{mV}$. Similarly, in $\mathrm{Ca}^{2+}$ imaging, the event duration is remarkably decreased or the pacemaker activity inhibited due to STIM1/SARAF, compared that of 
A

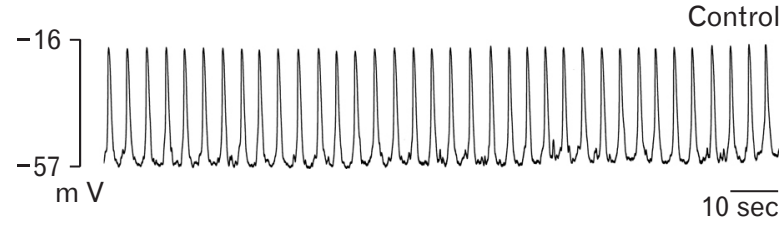

B

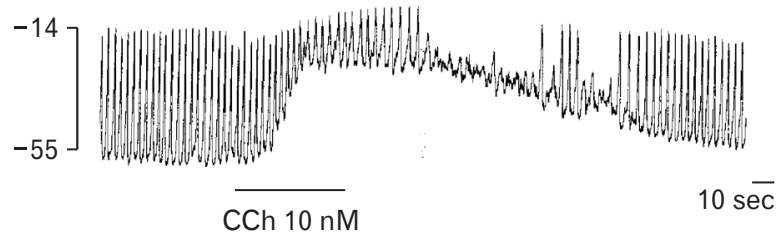

D

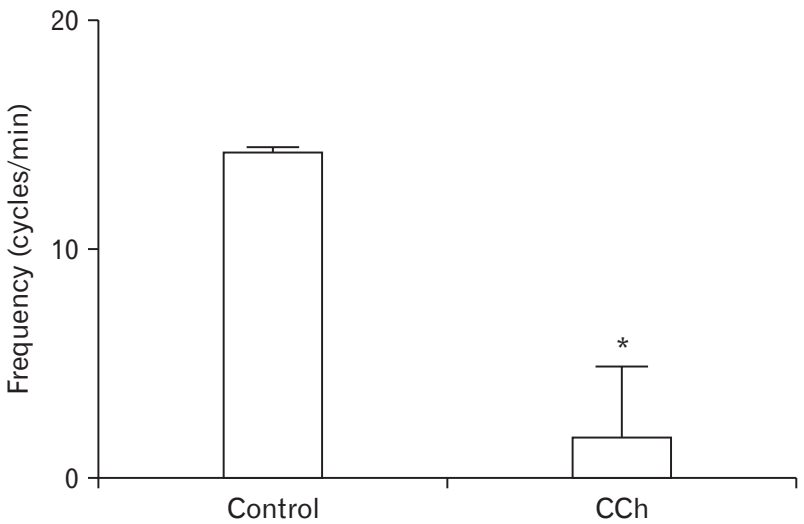

Figure. Effects of carbachol (CCh) treatment on pacemaker potentials recorded in cultured interstitial cells of Cajal (ICCs) from mouse small intestine. (A) Pacemaker potentials of ICCs in current clamping mode $(\mathrm{I}=0)$. The vertical solid lines represent the scale of pacemaker potential amplitudes, and the horizontal solid lines represent the scale of pacemaker potential recording duration. (B) Pacemaker potentials of ICCs exposed to $\mathrm{CCh}(10 \mathrm{nM})$ in the current clamping mode $(\mathrm{I}=0)$. Cellular responses to $\mathrm{CCh}$ are summarized in $(\mathrm{C})$ and $(\mathrm{D})$. The bars represent mean values \pm SE. Asterisks represent significant differences from untreated control cells (Control) $\left({ }^{\star} P<0.01\right)$. RMP, resting membrane potentials. Adapted from Park et al. ${ }^{1}$

control (empty cDNA transfection). This information suggests the endoplasmic reticulum proteins (STIM1/SARAF) may involve in pacemaker activity in cultured ICC, but it is an impairing effect.

It is curious that along with several baselines from the trace of either electrical or $\mathrm{Ca}^{2+}$ imaging from ICC treated by STIM1 and SARAF, there are lack of the modulations like $\mathrm{CCh}, \mathrm{Ca}^{2+}$ free and thapsigargin etc which are performed in non-treated ICC as presented in Figure. Of note molecular expressions of STIM1 and SARAF, the observation is screened in normal cultured condition, but not in overexpressional cultures. Without a parallel comparison about transfections on how much "overexpression" of such molecules in treatment versus to control weakens the "role of ER proteins STIM1/SARAF on pacemaker activity, and this novel study to understand how much is overexpression and the effects whether increase or decrease on ICC pacemaker activity are expected to be followed up.

Jian-Lin LV, ${ }^{1,2}$ Ning-Yuan Chen, ${ }^{1}$ and Shang-Ling Pan ${ }^{1}$ 'Department of Pathophysiology, School of Preclinical Medicine, Guangxi Medical University, Nanning; Guangxi, China; and ${ }^{2}$ The First Affiliated Hospital of Guangxi University of Chinese Medicine, Nanning, Guangxi, China
1. Park CG, Wu MJ, Hong C, et al. Regulation of intracellular calcium by endoplasmic reticulum proteins in small intestinal interstitial cells of Cajal. J Neurogastroenterol Motil 2018;24:128-137.

2. Koh SD, Sanders KM, Ward SM. Spontaneous electrical rhythmicity in cultured interstitial cells of Cajal from the murine small intestine. J Physiol 1998;513(Pt 1):203-213.

3. Huizinga JD, Zhu Y, Ye J, Molleman A. High-conductance chloride channels generate pacemaker currents in interstitial cells of Cajal. Gastroenterology 2002;123:1627-1636.

4. Kim TW, Koh SD, Ordög T, Ward SM, Sanders KM. Muscarinic regulation of pacemaker frequency in murine gastric interstitial cells of Cajal. J Physiol 2003;546:415-425.

5. Zhu MH, Sung IK, Zheng $\mathrm{H}$, et al. Muscarinic activation of $\mathrm{Ca}^{2+}$ activated $\mathrm{Cl}^{-}$current in interstitial cells of Cajal. J Physiol 2011;589(Pt 18):4565-4582.

6. Torihashi S, Fujimoto T, Trost C, Nakayama S. Calcium oscillation linked to pacemaking of interstitial cells of Cajal: requirement of calcium influx and localization of TRP4 in caveolae. J Biol Chem 2002;277:1919119197.

7. So KY, Kim SH, Sohn HM, et al. Carbachol regulates pacemaker activities in cultured interstitial cells of Cajal from the mouse small intestine. Mol Cells 2009;27:525-531.

8. Bayguinov PO, Hennig GW, Smith TK. $\mathrm{Ca}^{2+}$ imaging of activity in 
ICC-MY during local mucosal reflexes and the colonic migrating motor complex in the murine large intestine. J Physiol 2010;588(Pt 22):44534474.
Financial support: None.

Conflicts of interest: None.

Author contributions: Jianlin LV wrote the draft; Ning-Yuan Chen made a revision; and Shang-Ling Pan as corresponding author made the final revisions and corrections on the manuscript. 